\title{
Prinzipien zu offenen bibliographischen Daten $^{1}$
}

\section{Einleitung}

Produzenten bibliographischer Daten wie Bibliotheken, Verlage, Universitäten, Wissenschaftler oder soziale, webbasierte Literaturverwaltungsplattformen spielen eine wichtige Rolle bei der Entwicklung menschlichen Wissens. Damit ihre Arbeit der Gesellschaft in vollem Umfang zugutekommt, ist es zwingend erforderlich, bibliographische Daten zu öffnen, das heißt für alle zur beliebigen Nutzung frei verfügbar zu machen.

\section{Bibliographische Daten}

Um den Geltungsbereich der Prinzipien festzulegen, wird in diesem ersten Teil der zugrundeliegende Begriff bibliographischer Daten erläutert.

\section{Kerndaten}

Bibliographische Daten bestehen aus bibliographischen Beschreibungen. Eine bibliographische Beschreibung beschreibt eine bibliographische Ressource (Artikel, Monographie etc. - ob gedruckt oder elektronisch) zum Zwecke

1. der Identifikation der beschriebenen Ressource, d.h. des Zeigens auf eine bestimmte Ressource in der Gesamtheit aller bibliographischen Ressourcen und

2. der Lokalisierung der beschriebenen Ressource, d.h. eines Hinweises, wo die beschriebene Ressource aufzufinden ist.

1 Zur englischen Originalversion (zugänglich unter http://openbiblio.net/principles/) haben unter anderen beigetragen: Karen Coyle, Mark MacGillivray, Peter Murray-Rust, Ben O’ Steen, Jim Pitman, Adrian Pohl, Rufus Pollock, William Waites. Die Originalversion wurde am 3. Februar 2011 publiziert. Die deutsche Übersetzung stammt von Adrian Pohl und wurde am 3. Februar 2011 veröffentlicht. Lizenziert ist der Text der Übersetzung unter der CCO Public Domain Dedication (http://creativecommons.org/publicdomain/zero/1.0/). 
Traditionellerweise erfüllte eine Beschreibung beide Zwecke gleichzeitig, indem sie Information lieferte über: Autor(en) und Herausgeber, Titel, Verlag, Veröffentlichungsdatum und -ort, Identifizierung des übergeordneten Werks (z.B. einer Zeitschrift), Seitenangaben.

Im Web findet Identifikation statt mittels Uniform Resource Identifiers (URIs) wie z.B. URNs oder DOIs. Lokalisierung wird durch HTTP-URIs ermöglicht, die auch als Uniform Resource Locators (URLs) bezeichnet werden. Alle URIs für bibliographische Ressourcen fallen folglich unter den engen Begriff bibliographischer Daten.

\section{Sekundäre Daten}

Eine bibliographische Beschreibung kann andere Informationen enthalten, die unter den Begriff bibliographischer Daten fallen, beispielsweise Nicht-WebIdentifikatoren (ISBN, LCCN, OCLC etc.), Angaben zum Urheberrechtsstatus, administrative Daten und mehr; diese Daten können von Bibliotheken, Verlagen, Wissenschaftlern, Online-Communities für Buchliebhaber, sozialen Literaturverwaltungssystemen und anderen produziert sein.

Darüber hinaus produzieren Bibliotheken und verwandte Institutionen kontrollierte Vokabulare zum Zwecke der bibliographischen Beschreibung wie z. B. Personen- und Schlagwortnormdateien, Klassifikationen etc., die ebenfalls unter den Begriff bibliographischer Daten fallen.

\section{Vier Prinzipien}

Wir empfehlen ausdrücklich die Übernahme und Umsetzung der folgenden Prinzipien:

1. Wenn bibliographische Daten oder Sammlungen bibliographischer Daten veröffentlicht werden, sind die Wünsche und Erwartungen des Herausgebers im Hinblick auf Fragen der Nachnutzung und Wiederverwendung einzelner bibliographischer Beschreibungen, der gesamten Sammlung sowie Teilmengen der Sammlung klar und explizit anzugeben. Diese Angabe sollte präzise und unwiderruflich sein sowie auf einer angemessenen und anerkannten rechtlichen Erklärung in der Form eines „Waivers“ (Verzichtserklärung) oder einer Lizenz basieren.

Die Veröffentlichung bibliographischer Daten erfolge mit einer expliziten und tragfähigen rechtlichen Erklärung. 
2. Viele weithin anerkannte Lizenzen sind weder bestimmt noch geeignet für bibliographische Daten oder Sammlungen bibliographischer Daten. Eine Auswahl von „Waivern“ und Lizenzen, die für die Anwendung auf Daten konzipiert und geeignet sind, ist beschrieben unter http://opendefinition. org/licenses/\#Data. Creative-Commons-Lizenzen (mit Ausnahme der CCO), GFDL, GPL, BSD etc. sind NICHT geeignet für Daten und von ihrer Nutzung wird DRINGEND abgeraten.

Benutze eine für Daten geeignete anerkannte Lizenz oder einen Waiver.

3. Von der Benutzung von Lizenzen, die eine kommerzielle Nachnutzung verhindern oder eine Produktion derivativer Werke beschränken, indem die Nutzung für bestimmte Zwecke oder durch bestimmte Personen oder Organisationen ausgeschlossen wird, wird DRINGEND abgeraten. Solche Lizenzen machen es unmöglich, Datenbestände effektiv zu integrieren und nachzunutzen. Außerdem verhindern sie die Entstehung kommerzieller Dienste, die eine Aufwertung der Daten leisten sowie kommerzieller Aktivitäten, die zur Erhaltung der Datenmengen beitragen können.

Um eine effektive Nutzung und Verbesserung der Daten durch andere zu erreichen, sollten die Daten offen im Sinne der Open Definition (http:// opendefinition.org/) sein - insbesondere der Ausschluss kommerzieller Nutzung sowie andere einschränkende Klauseln sollten unterlassen werden.

4. Darüber hinaus empfehlen wir - insbesondere öffentlich finanzierte - bibliographische Daten und Sammlungen bibliographischer Daten explizit in die Public Domain durch die Nutzung der Public Domain Dedication and Licence oder des Creative Commons Zero Waivers zu geben. Dadurch werden die Nachnutzungsmöglichkeiten maximiert, ganz im Sinne des allgemeinen Ethos des Teilens im Bereich öffentlich geförderter Gedächtnisinstitutionen.

Wo möglich, empfehlen wir, bibliographische Daten mittels PDDL oder CCo explizit in die Public Domain zu geben.

\section{Addendum}

Eine unvollständige Liste bibliographischer Daten.

Kerndaten: Namen und Identifikatoren von Autor(en) und Herausgeber(n), Titel, Verlagsinformation, Veröffentlichungsdatum und -ort, Identifizierung des übergeordneten Werks (z.B. einer Zeitschrift), Seitenangaben, URIs.

Sekundäre Daten: Formatangaben, Nicht-Web-Identifikatoren (ISBN, LCCN, OCLC-Nummer etc.), Angaben zu Urheber- und Lizenzstatus, Angaben finanzieller Förderer, Angaben zum Trägermedium, Umfang- und Größenangaben, admi- 
nistrative Daten (letzte Änderung des Datensatzes etc.), relevante Links (zu Wikipedia, Google Books, Amazon etc.), Inhaltsverzeichnis, Links zu digitalisierten Auszügen eines Textes (Inhaltsverzeichnis, Register, Literaturverzeichnis etc.), Adresse und andere Kontaktdetails zum Autor/den Autoren, Coverabbildungen, Abstracts, Rezensionen, Zusammenfassungen, Schlagwörter, Stichwörter, Notationen, nutzergenerierte Tags, Exemplardaten (Signatur etc.). 